\title{
Identification of Functional Thyroid Stimulating Hormone Receptor and TSHR Gene Mutations in Hepatocellular Carcinoma
}

\author{
YU-LIN SHIH ${ }^{1,2}$, YA-HUI HUANG ${ }^{1}$, KWANG-HUEI LIN $^{1,3}$, YU-DE CHU $^{1}$ and CHAU-TING YEH ${ }^{1,2}$ \\ ${ }^{1}$ Liver Research Center, Chang Gung Memorial Hospital, Taoyuan, Taiwan, R.O.C.; \\ ${ }^{2}$ Molecular Medicine Research Center, Chang Gung University, Taoyuan, Taiwan, R.O.C.; \\ ${ }^{3}$ Department of Biochemistry, School of Medicine, Chang Gung University, Taoyuan, Taiwan, R.O.C.
}

\begin{abstract}
Background/Aim: Extra-thyroid expression of thyroid stimulating hormone (TSH) receptor (TSHR) has been reported in normal liver tissues, but never assessed in hepatocellular carcinoma (HCC). Patients and Methods: Paired cancerous and non-cancerous HCC tissues were analyzed with TSHR expression assays. TSHR functional assessments and sequence analysis for the TSHR exon-10 were performed. Results: TSHR overexpression was found in 150/197 (76.1\%) HCCs. Higher TSHR expression was associated with unfavorable postoperative outcomes. Immunohistochemical analysis revealed predominantly nuclei/peri-nuclei localization of TSHR in cancerous tissues but cell membrane localization in non-cancerous parts. TSH stimulation on hepatoma cells resulted in increased cyclic adenosine monophosphate levels with altered cell sensitivity to cisplatin. Gene mutations leading to TSHR truncation were detected in $8 / 81$ (9.9\%) HCC tissues. Conclusion: Overexpression of TSHR was found in a great majority of HCC tissues and associated with unfavorable prognosis. Cell-based experiments and gene mutation analysis suggested that TSHR in HCCs was functional.
\end{abstract}

Globally, hepatocellular carcinoma (HCC) ranks as the sixth most common solid tumor and the third leading cause of cancer-related mortality (1). Major causes of HCC include

Correspondence to: Chau-Ting Yeh, Director, Liver Research Center, Chang Gung Memorial Hospital, 5 Fu-Shin Street, KueiShan District, Taoyuan, Taiwan, R.O.C. Tel: +886 33281200 ext. 8129, Fax: +886 33282824, e-mail: chautingy@gmail.com or YaHui Huang, Liver Research Center, Chang Gung Memorial Hospital, 5 Fu-Shin Street, Kuei-Shan District, Taoyuan, Taiwan, R.O.C. Tel: +88633281200 ext. 8129 , Fax: +88633282824 , e-mail: e1249060@gmail.com

Key Words: Thyroxine, thyroid stimulating hormone, hepatocellular carcinoma. viral hepatitis, alcohol abuse, non-alcoholic steatohepatitis, diabetes, aflatoxin, and cirrhosis (2). HCC can be treated by being completely removed/eradicated, with a liver transplantation, transcatheter arterial chemoembolization, or with the use of targeted agents or systemic chemotherapy, depending on the clinical stage of the disease (3-5). Owing to the asymptomatic nature of this disease, a great majority of patients $(>50 \%)$ are diagnosed in the intermediate and advanced stages in Taiwan (6).

Thyroid hormone plays an essential role in the growth, maturation, differentiation and metabolism of normal cells (7). Additionally, several small-scale clinical studies as well as animal studies have shown that thyroid hormone can promote cell growth of malignant tumors (8-10). In two clinical trials for glioblastoma multiforme, medically induced hypothyroidism was associated with significantly longer progression-free survival (11). In metastatic renal cancer patients, hypothyroidism developed during sorafenib or sunitinib treatment was correlated with a better prognosis (12). Similar observation has also been made for head and neck cancer (13). In HCC, however, such studies have not been conducted. Notably, several epidemiological and animal studies argued against the possible beneficial effect of hypothyroidism in HCC. Hypothyroidism, on the contrary, has been proposed to be a possible risk factor for liver cancer $(14,15)$. Furthermore, our previous studies showed that thyroid hormone up-regulated the expression of dickkopf 4 , a putative tumor suppressor gene, and suppressed cancer cell proliferation. It also down-regulated the expression of pituitary tumor transforming gene 1 , a putative oncogene, in a subset of HCC specimens $(16,17)$. Other studies also supported its role in growth suppression of $\operatorname{HCC}(18,19)$.

Another yet complicated issue when studying clinical outcomes of cancer patients with hypothyroidism is that these patients usually harbor an elevated level of thyroid stimulating hormone (TSH). Physiologically, there is a negative feedback mechanism where the pituitary secretes additional TSH when 
the thyroid hormone level is low, so that the thyroid gland can produce more thyroid hormone. Several previous studies have identified TSH receptor (TSHR) expression in human extrathyroid tissues or cells, including adipocytes $(20,21)$, osteocytes (22), erythrocytes (23), cardiac muscle (24), thymus (25) and normal liver (26). Furthermore, TSHR has also been detected in several extra-thyroid cancers, including lung cancer (27), breast cancer (28), and ovarian cancer (29). Conceivably, elevated TSH levels should affect the physiological functions of these cells. However, it has not been well explored whether TSHR-mediated signaling could alter cancer cell growth or affect cancer cell sensitivity to cytotoxic agents. Additionally, it has not been demonstrated whether TSHR is expressed in human HCC tissues.

In this study, we examined TSHR expression in noncancerous and cancerous parts of surgically removed HCC tissues and correlated it with postoperative outcomes. TSHR function was illustrated in HCC cell lines under TSH stimulation. Mutations were detected in the previously reported exon-10 hot area of the TSHR gene.

\section{Patients and Methods}

Patients. This study was conducted under the approval of the Institutional Review Board, Chang Gung Memorial Hospital. All patients had signed an informed consent form before deposition of their samples to Tissue bank, Linko Chang Gung Medical Center. Totally, 197 paired HCC tissues (cancerous and adjacent noncancerous parts) were retrieved from the tissue bank for TSHR expression analysis. Clinical data were collected for outcome analysis, including gender, age, hepatitis B virus surface antigen (HBsAg), anti-hepatitis $\mathrm{C}$ virus antibody (anti-HCV), liver cirrhosis, alcohol abuse, microvascular invasion, macrovascular invasion, ascites, histology grade, capsule, tumor number, largest tumor size (in diameter), alpha-fetoprotein (AFP), albumin, bilirubin, prothrombin time, aspartate transaminase (AST), alanine transaminase (ALT), and creatinine level.

Immunohistochemistry and western blot analysis. Immunohistochemistry and western blot analysis were performed using rabbit anti-TSHR polyclonal antibody (Bioss Antibodies Inc., Woburn, MA, USA). Details of immunohistochemical analysis were described in our previous publication (30). Briefly, liver tissues were fixed in $10 \%$ formaldehyde and embedded in paraffin. Fivemicrometer paraffin sections were mounted on poly-L-lysine-coated slides. After deparaffinization in xylene, the sections were rehydrated through graded ethanol. TSHR expression was assessed by the avidin-biotin immunoperoxidase method. The sections were incubated in PBS $(0.1 \mathrm{~mol} / \mathrm{l}$ [pH 7.4]) containing 5\% hydrogen peroxide for $15 \mathrm{~min}$ and washed twice $(5 \mathrm{~min}$ each) in PBS containing $0.025 \%$ Triton X-100 (Sigma Chemical, Saint Louis, MO, USA). The tissue sections were then incubated with $3 \%$ bovine serum albumin and $10 \%$ normal goat serum for $30 \mathrm{~min}$, followed by a 1:500 dilution of rabbit anti-TSHR polyclonal antibody at $37^{\circ} \mathrm{C}$ for $1 \mathrm{~h}$. After being washed with PBS, sections were incubated with a 1:400 dilution of biotin-conjugated goat anti-rabbit immunoglobulins (Jackson ImmunoResearch, West Grove, PA,
USA) for $40 \mathrm{~min}$. After being rinsed with PBS, sections were treated with avidin-biotin complex (Vectastain Elite ABC Kit; Vector Laboratories, Burlingame, CA, USA) for $30 \mathrm{~min}$, and the reaction products were visualized in diaminobenzidine solution (Vector Laboratories, Burlingame, CA, USA). Counterstaining was performed using hematoxylin.

To assess TSHR expression, $10 \mu \mathrm{g}$ of protein extract obtained from liver tissues were loaded onto a polyacrylamide gel for electrophoresis and then transferred to a nitrocellulose membrane. Western blot analysis was performed using rabbit anti-TSHR antibody (1: 2000 dilution) with goat anti-rabbit horseradish peroxidase conjugated antibody (Millipore Corp., Bedford, MA, USA) as the secondary antibody. GAPDH was detected by the use of mouse antiGAPDH antibody (1: 20000 dilution; Proteintech.) with goat antimouse horseradish peroxidase conjugated antibody (Millipore Corp., Bedford, MA, USA) as the secondary antibody. In each western blot, the same amount of HeLa whole cell lysate was loaded onto the rightmost lane to serve as a positive control as suggested by the manufacturer. The intensities of immunoreactive bands were quantified using Image Gauge software (Fuji Film, Tokyo, Japan). All density readings were normalized with the corresponding GAPDH levels. After normalization, the cancerous/noncancerous ratios (T/N ratios) of TSHR were calculated accordingly. To correlate the (cancerous or noncancerous) TSHR levels and postoperative outcomes, the mean density values of normalized TSHR levels in the cancerous and noncancerous parts, respectively, were assigned as 100 Hepatic Unit (HU) before performing statistical analysis.

Cell culture. Details of maintenance and culture of Huh7, J7, Hep3B, Alexander and Mahlavu cells were described in our previous publications $(31,32)$. HepY1 and HepY2 HCC cell lines were established in our laboratory (32).

TSH stimulation assay. TSH-free medium was generated by replacing bovine serum with TSH-free serum (BBI Solutions, Cardiff, UK). Cells were maintained in TSH-free medium for $24 \mathrm{~h}$. TSH (Thyrotropin alfa, Sanofi Taiwan Co., Ltd, Taiwan) was added, at the desirable concentration, for $1 \mathrm{~h}$. Cellular cyclic adenosine monophosphate (cAMP) was measured using cAMP Screen Assay System (Thermo Fisher Scientific, Waltham, MA, USA) according to the manufacturer's protocol.

Detection of TSHR gene mutations in exon-10. Chromosomal DNA was extracted from non-cancerous and cancerous parts of HCC tissues according to our previous protocol (32). DNA fragment flanked by the primers TSHR-U, 5'-AATTGTGGTGTGGTT CGTTAG-3' (nt. 1410 to 1430; GenBank reference sequence NM_000369) and TSHR-D, 5'- TTTACAGATGCCAAACTTGC-3' (nt. 2256 to 2237) was amplified by PCR using the program consisting of $94^{\circ} \mathrm{C}(1 \mathrm{~min}), 55^{\circ} \mathrm{C}(1 \mathrm{~min})$ and $72^{\circ} \mathrm{C}(1 \mathrm{~min})$ for 30 cycles in a thermal cycler (Perkin-Elmer Cetus, Norwalk, CT, USA). The amplicon was sent for nucleotide sequence analysis from both directions.

\section{Results}

Expression of TSHR in HCCs. To examine whether TSHR was expressed in human HCCs, 197 paired HCC tissues were submitted for immunohistochemistry and western blot analysis (Figure 1). The baseline clinical data are listed in 
A

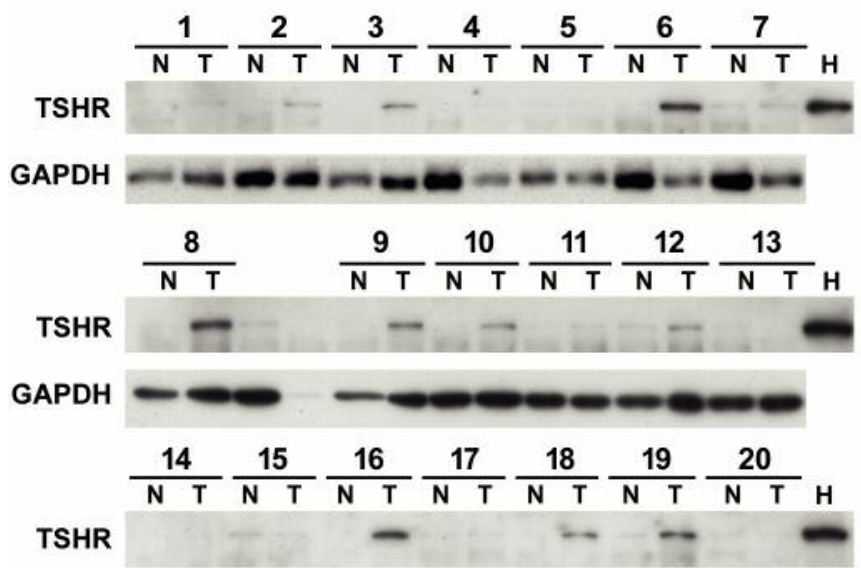

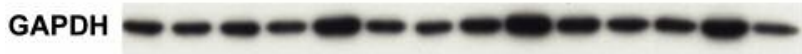

$\frac{21}{N T} \frac{22}{N T} \frac{23}{N T} \frac{24}{N T} \frac{25}{N T T} \frac{26}{N T T} \frac{27}{N T H}$

TSHR

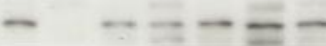

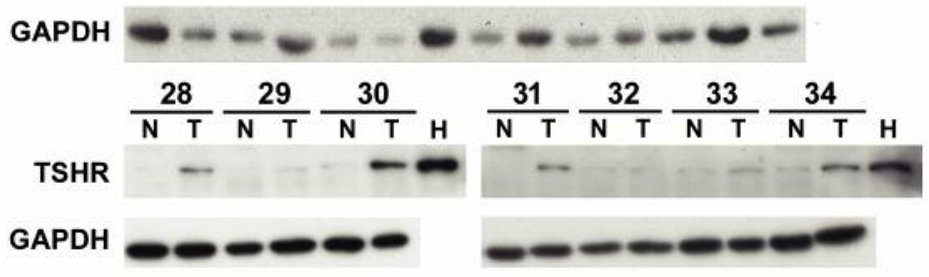

B

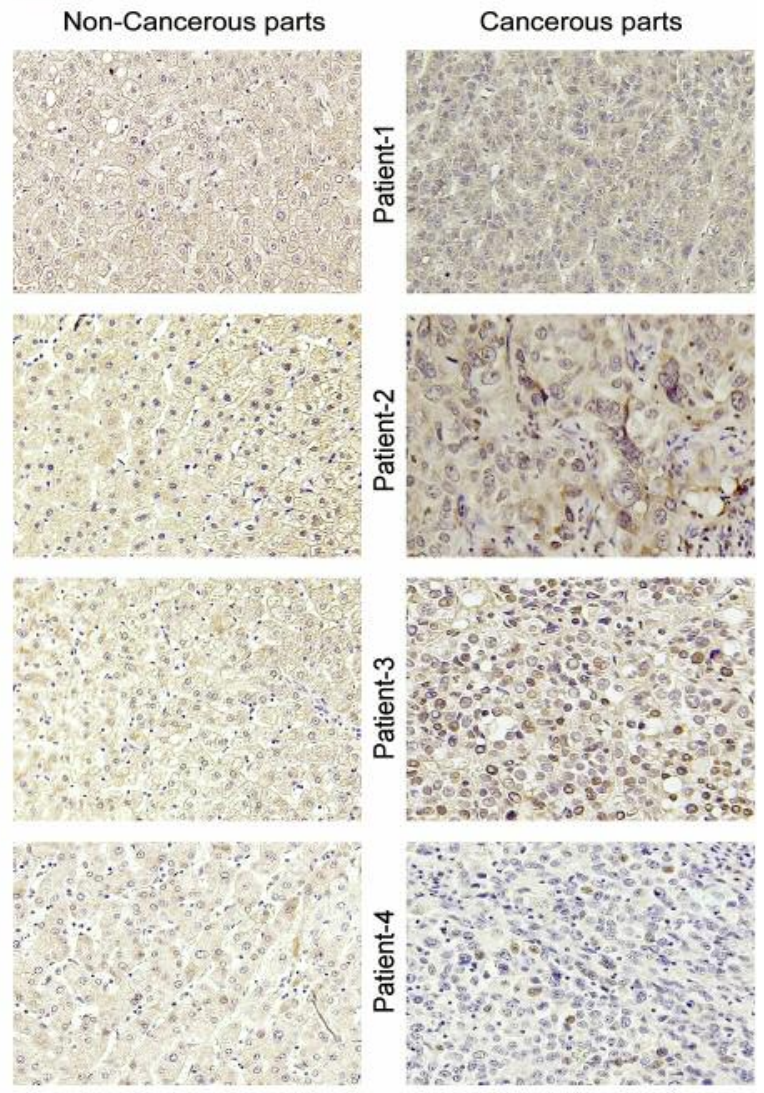

Figure 1. Expression of TSHR in HCC. (A) Western blot analysis of TSHR expression in paired HCC tissues. N: noncancerous tissue; T: cancerous tissues; GAPDH: as loading controls. H: protein lysates from HeLa cells, as positive controls for TSHR. Molecular weight of reactive bands was $115 \mathrm{kDa}$. (B) Immunohistochemical analysis of TSHR expression in HCC tissues. Left panels: noncancerous tissues; right panels: cancerous tissues.

Table I. It was found that TSHR was expressed in both cancerous and noncancerous parts of HCC tissues. Western blot analysis showed that TSHR overexpression $(\mathrm{T} / \mathrm{N}$ ratio of TSHR >1) was found in 150/197 (76.1\%) patients (Figure 1A). Immunohistochemical analysis revealed that in the noncancerous parts, TSHR was primarily located in the cell membrane (Patient-1 to 3), but could also be detected in the cytosol (Patient-1 to 4). In the cancerous parts, however, it was located in the cytosol (Patient-1), peri-nuclear area (Patient-2), and nucleus (Patient-3 and -4) (Figure 1B). Expression levels, measured by densitometry using a western blot film, were correlated with postoperative outcomes. It was found that a higher expression of TSHR in the noncancerous parts was correlated with an unfavorable intrahepatic recurrence-free survival ( $p=0.032$; Table II; Cox proportional hazard analysis). Multivariate analysis indicated that TSHR expression in noncancerous parts was an independent predictor of intrahepatic recurrence-free survival (adjusted $p=0.012$ ). Kaplan-Meier analysis also showed the same result (Figure 2A, Log-rank $p=0.031$ ). On the other hand, higher expression of TSHR in cancerous parts and higher $\mathrm{T} / \mathrm{N}$ ratio of TSHR were both correlated with unfavorable metastasis-free survival $(p=0.048$ and 0.036 , respectively; Table III; Cox proportional hazard analysis). Kaplan-Meier analysis showed the same results (Figure 2B and 2C; Log-rank $p=0.038$ and 0.030 , respectively). However, multivariate analysis indicated that these two factors were not independent predictors of metastasis-free survival. Linear regression analysis showed that TSHR expression in HCC cancerous parts was independently associated with a higher histology grade $(p=0.048)$ and AFP level $(p=0.001)$ (Table IV).

TSH stimulation resulted in elevated cAMP in HCC cells. To understand whether TSHR has a function in HCC cells, a TSH stimulation assay was performed (Figure 2D-F). TSHR expression was first confirmed by immunocytochemistry staining (Figure 2D) in Huh7 and J7 cells. 
Table I. Basic clinicopathological data of 197 HCC patients receiving surgical resection.

\begin{tabular}{lc}
\hline Parameters & \\
\hline Gender, Male & $157(79.7 \%)$ \\
Age, years & $55.4 \pm 14.0$ \\
HBsAg, positive & $150(76.1 \%)$ \\
Anti-HCV, positive & $55(27.9 \%)$ \\
Cirrhosis, Yes & $127(64.5 \%)$ \\
Alcoholism, Yes & $49(24.9 \%)$ \\
Microvascular invasion, Yes & $63(32.0 \%)$ \\
Macrovascular invasion, Yes & $32(16.2 \%)$ \\
Ascites, Yes & $17(8.6 \%)$ \\
Histology grade & \\
1 & $4(2.0 \%)$ \\
2 & $56(28.4 \%)$ \\
3 & $116(58.9 \%)$ \\
4 & $21(10.7 \%)$ \\
Capsule, Yes & $145(73.6 \%)$ \\
Tumor number, & \\
1 & $96(48.7 \%)$ \\
2 & $56(28.4 \%)$ \\
3 & $30(15.2 \%)$ \\
$>3$ & $15(7.6 \%)$ \\
Tumor size, cm & $5.6 \pm 3.9$ \\
Biochemistry & \\
AFP, ng/ml & \\
Albumin, g/dl & $37.25(1.86$ to 443209$)$ \\
Bilirubin, mg/dl & $3.9 \pm 0.6$ \\
Prothrombin time, sec & $1.2 \pm 1.5$ \\
AST, U/l & $12.2 \pm 1.5$ \\
ALT, U/l & $60.4 \pm 89.5$ \\
Creatinine, mg/dl & $72.6 \pm 132.5$ \\
\hline & $1.2 \pm 1.0$ \\
\hline
\end{tabular}

TSH stimulation was performed using a range of different concentrations. It was found that in both Huh7 and J7 cells, intracellular cAMP increased in a dose-dependent manner (Figure 2E-F). A high concentration of TSH was required to elicit a cAMP response in $\mathrm{J} 7$ cells. To understand whether this was a nonspecific response, we used a negative control using HEK293 cells, in which TSHR was absent (33). No cAMP elevation was observed under the stimulation with the highest level of TSH as in the case of $\mathbf{J} 7$ cells (data not shown).

Altered sensitivity to cisplatin upon TSH stimulation in HCC cells. TSHR was detected in $8 \mathrm{HCC}$ cell lines (Figure 3A). Of them, 5 HCC cell lines, Hep3B, Huh7, J7, Alexander, and Mahlavu, were cultured in TSH-free medium with or without the addition of TSH (in a concentration of $8 \mathrm{mIU} / \mathrm{l}$ ) for $24 \mathrm{~h}$. Cells were treated with various concentrations of cisplatin for $24 \mathrm{~h}$ and cell viability was assessed using 3(4,5-Dimethylthiazol-2-yl)-2,5-diphenyltetrazolium bromide (MTT) assay. It was found that TSH mildly enhanced the cell
Table II. Univariate and multivariate cox proportional hazard analysis for time-to-intrahepatic recurrence in HCC patients receiving surgical resection.

\begin{tabular}{lccc}
\hline Parameters & Hazard ratio & $95 \% \mathrm{CI}$ & $p$-Value \\
\hline Gender, Male & 1.329 & $0.795-2.221$ & 0.279 \\
Age, $>45$ years & 1.273 & $0.777-2.085$ & 0.339 \\
HBsAg, positive & 0.760 & $0.484-1.196$ & 0.236 \\
Anti-HCV, positive & 0.873 & $0.552-1.378$ & 0.559 \\
Cirrhosis, Yes & 1.144 & $0.745-1.757$ & 0.540 \\
Alcoholism, Yes & 1.110 & $0.698-1.767$ & 0.659 \\
Microvascular invasion, Yes & 1.497 & $0.979-2.387$ & 0.062 \\
Macrovascular invasion, Yes & 1.236 & $0.699-2.185$ & 0.466 \\
Ascites, Yes & 1.943 & $1.002-3.770$ & 0.049 \\
Histology grade, $>2$ & 1.159 & $0.754-1.782$ & 0.502 \\
Capsule, Yes & 1.330 & $0.831-2.129$ & 0.235 \\
Tumor number, $>1$ & 0.942 & $0.628-1.411$ & 0.771 \\
Tumor size, $>6$ cm & 1.759 & $1.145-2.702$ & 0.010 \\
Biochemistry & & & \\
AFP, $>30$ ng/ml & 1.442 & $0.959-2.167$ & 0.078 \\
Albumin, $>3.9 \mathrm{~g} / \mathrm{dl}$ & 0.613 & $0.408-0.920$ & 0.018 \\
Bilirubin, $>1.1 \mathrm{mg} / \mathrm{dl}$ & 0.983 & $0.605-1.598$ & 0.945 \\
Prothrombin time, $>12$ sec & 1.249 & $0.834-1.870$ & 0.280 \\
AST, $>39$ U/1 & 1.519 & $1.014-2.276$ & 0.043 \\
ALT, $>42$ U/1 & 1.040 & $0.694-1.560$ & 0.848 \\
Creatinine, $>1 \mathrm{mg} / \mathrm{dl}$ & 0.731 & $0.487-1.098$ & 0.132 \\
TSHR & & & \\
TSHR_N, $>110 \mathrm{HU}$ & 1.573 & $1.040-2.381$ & 0.032 \\
TSHR_T, $>115 \mathrm{HU}$ & 1.065 & $0.691-1.642$ & 0.776 \\
TSHR_T/N ratio, $>2.4$ & 0.788 & $0.525-1.183$ & 0.250 \\
\hline
\end{tabular}

Multivariate analysis (Entry mode)

\begin{tabular}{lccc}
\hline Parameters & Hazard ratio & 95\%CI & $p$-Value \\
\hline Ascites, Yes & 1.731 & $0.857-3.496$ & 0.126 \\
Tumor size, $>6 \mathrm{~cm}$ & 1.563 & $1.006-2.428$ & 0.047 \\
Albumin, $>3.9 \mathrm{~g} / \mathrm{dl}$ & 0.672 & $0.438-1.031$ & 0.069 \\
AST, $>39 \mathrm{U} / 1$ & 1.337 & $0.883-2.024$ & 0.170 \\
TSHR_N, $>110 \mathrm{HU}$ & 1.722 & $1.128-2.629$ & 0.012 \\
\hline
\end{tabular}

sensitivity to cisplatin in Hep3B and Huh7 cells, but greatly increased cell resistance in $\mathrm{J} 7$ and Alexander cells. No effect was observed in Mahlavu cells (Figure 3B).

Detection of TSHR gene mutations in exon-10. In several thyroid diseases, TSHR mutations were frequently detected in exon-10. We, thus, investigated whether there were gene mutations in exon 10 of TSHR in HCCs. In total, 150 noncancerous tissues and 81 cancerous tissues had sufficient amounts of DNA to be used in PCR-sequencing analysis. Only synonymous (silent) mutations could be found in the 150 noncancerous tissues; whereas mutations leading to a frame-shift or a premature stop codon were found in $8 / 81$ $(9.9 \%)$ of the cancerous tissues (Figure 4$)$. 
A

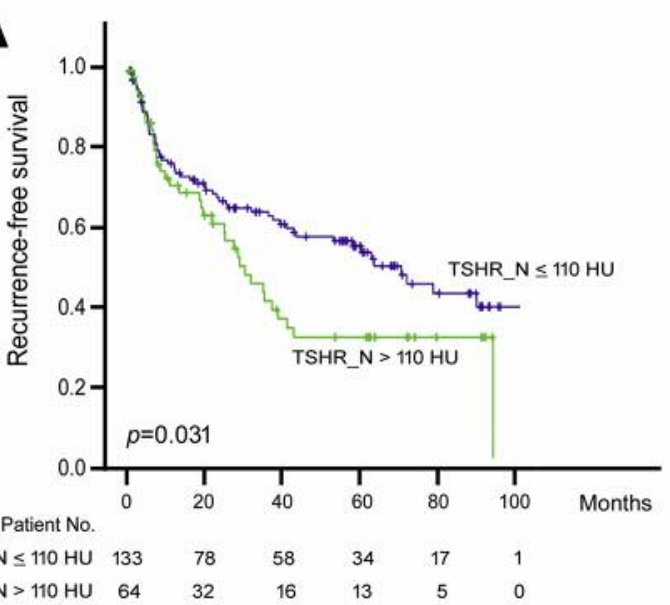

$\begin{array}{lrrrrrr}\text { TSHR_N } \leq 110 \mathrm{HU} & 133 & 78 & 58 & 34 & 17 & 1 \\ \text { TSHR_N > 110 HU } & 64 & 32 & 16 & 13 & 5 & 0\end{array}$

B

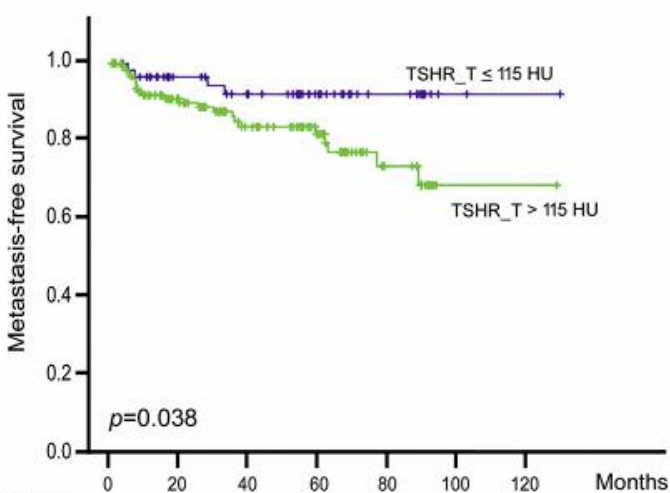

Patient No

$\begin{array}{lllllllll}\text { TSHR_T } \leq 115 \mathrm{HU} & 63 & 45 & 37 & 25 & 13 & 2 & 1\end{array}$

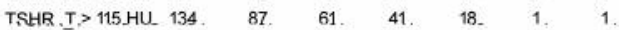

\section{C}

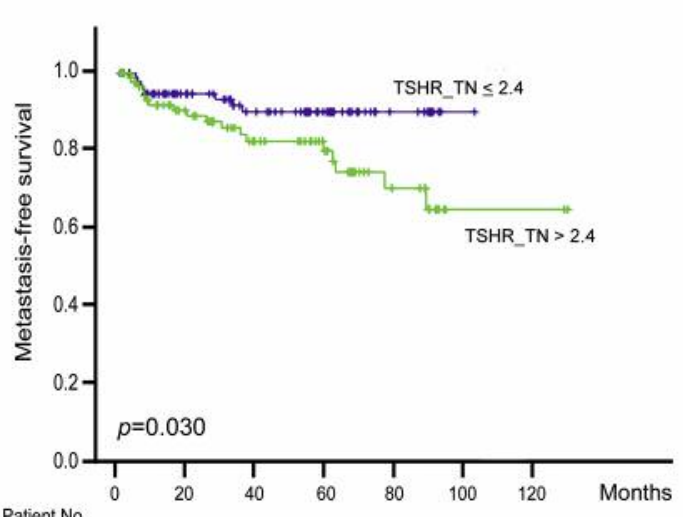
Patient No.

TSHR TN $\leq 2.4 \quad 102$

TSHR_TN $>2.4$
D

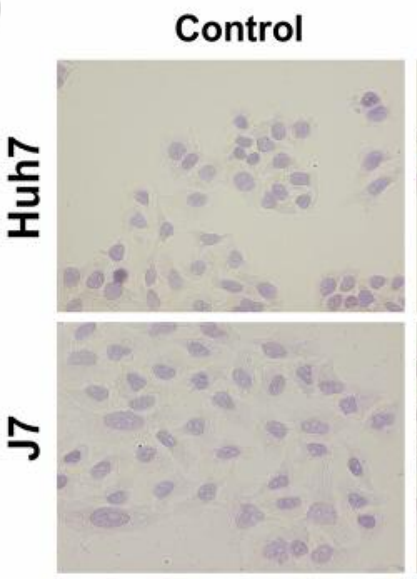

anti-TSHR

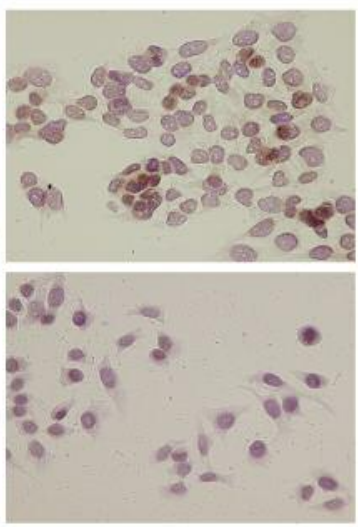

E

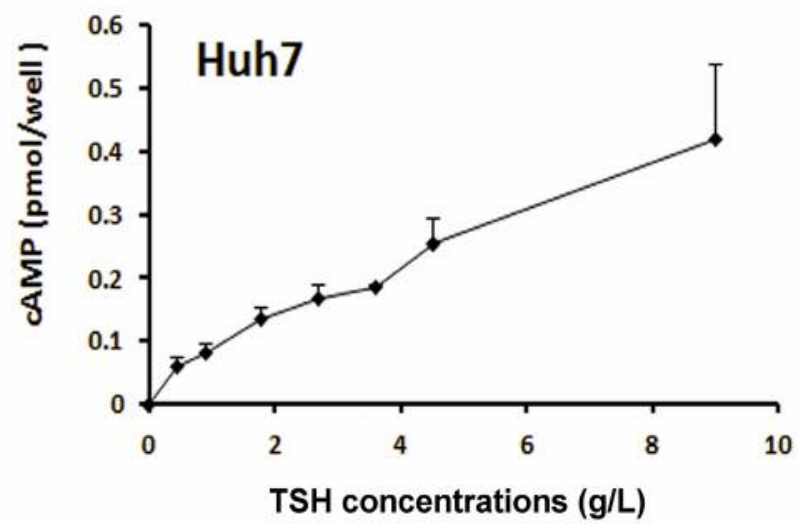

F

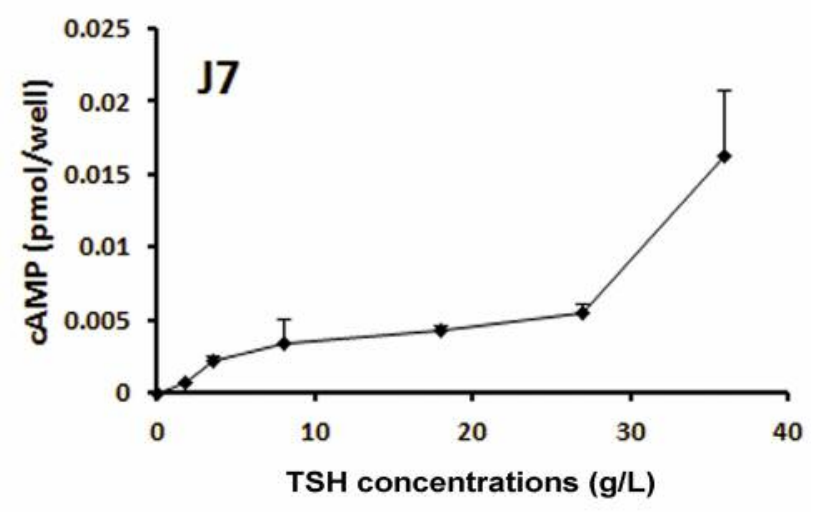

Figure 2. Postoperative prognosis of HCC patients receiving surgical resection (A to $C$ ) and TSH stimulation in HCC cells (D to F). (A) Kaplan-Meier analysis for intra-hepatic recurrence-free survival in HCC patients receiving surgical treatment in relationship to the abundance of TSHR in noncancerous tissue (TSHR_N); HU: fold increase of TSHR levels compared to the levels of TSHR in the cancerous tissues of patient-1 $\times 100$. (B and C) Kaplan-Meier analysis for extra-hepatic metastasis-free survival in HCC patients receiving surgical treatment in relationship to the abundance of TSHR in cancerous tissue (TSHR_T; B) or the cancerous/noncancerous ratio $\left(T S H R \_T N ; C\right)$. (D) Immunocytochemical analysis of TSHR expression in Huh7 (upper panels) or J7 (lower panels) cells. Cells were incubated with either preimmune serum (control) or anti-TSHR antibody. (E and F) Elevation of intracellular cAMP levels upon TSH stimulation in Huh7 (E) or J7 (F) cells. 
A

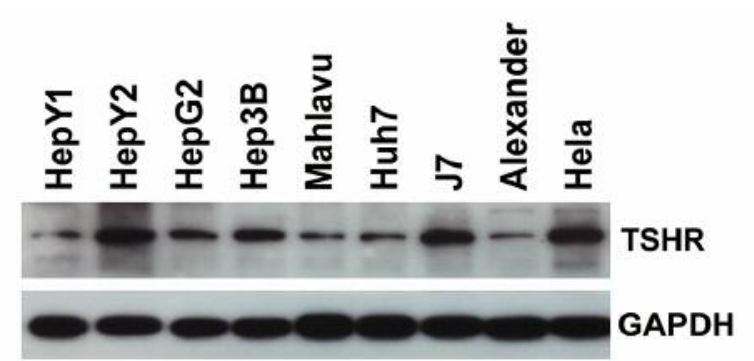

B
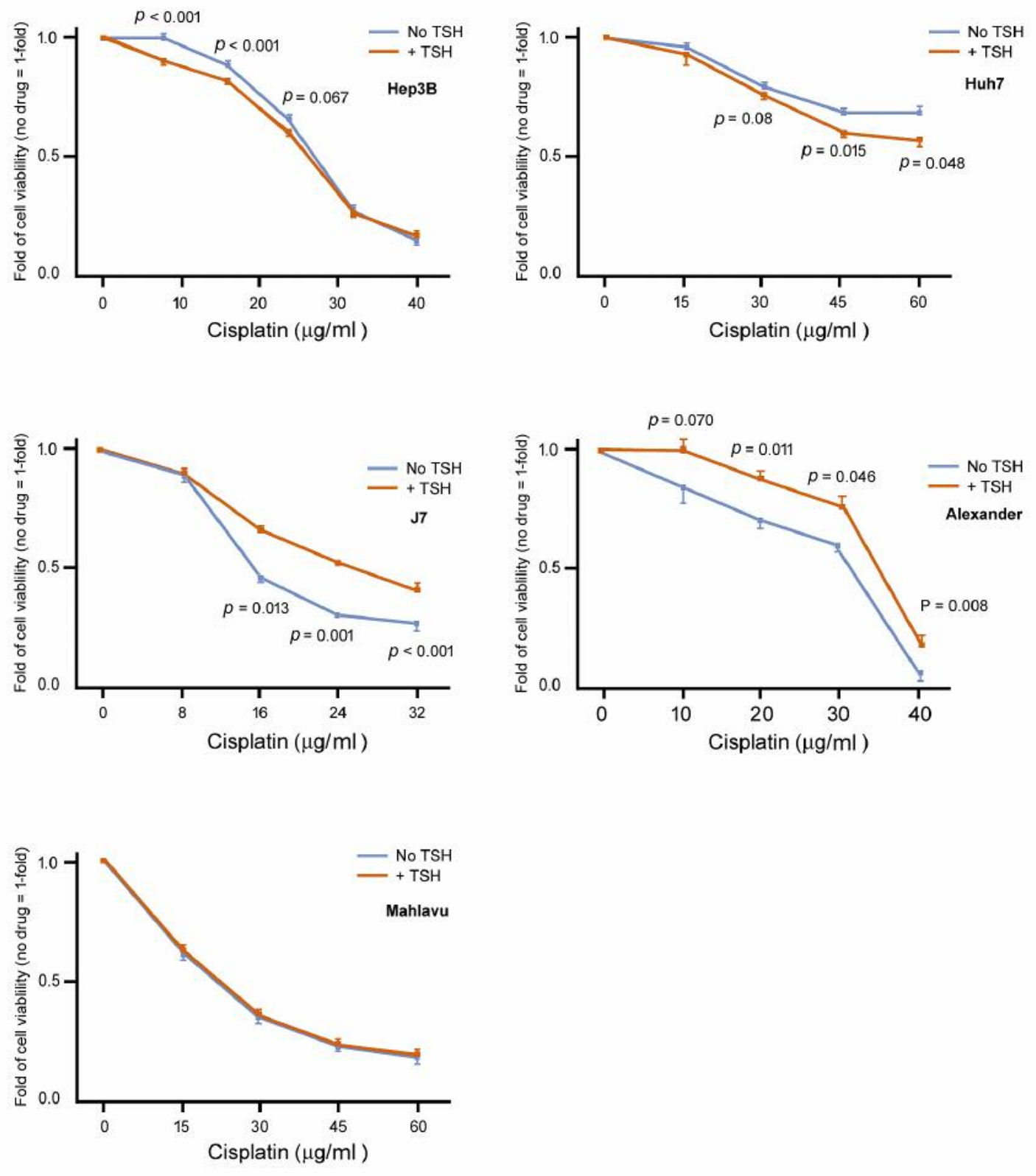

Figure 3. Alterations of cell sensitivity to cisplatin upon TSH stimulation. A: Western blot analysis was performed to illustrate expression of TSHR in all HCC cell lines tested. Molecular weight of reactive bands was $115 \mathrm{kDa}$. B: Alterations of cell sensitivity to cisplatin upon TSH stimulation in Hep3B (upper left), Huh7 (upper right), J7 (middle left), Alexander (middle right), and Mahlavu (lower) cells. Blue lines: no TSH treatment; Red lines: with TSH stimulation. 


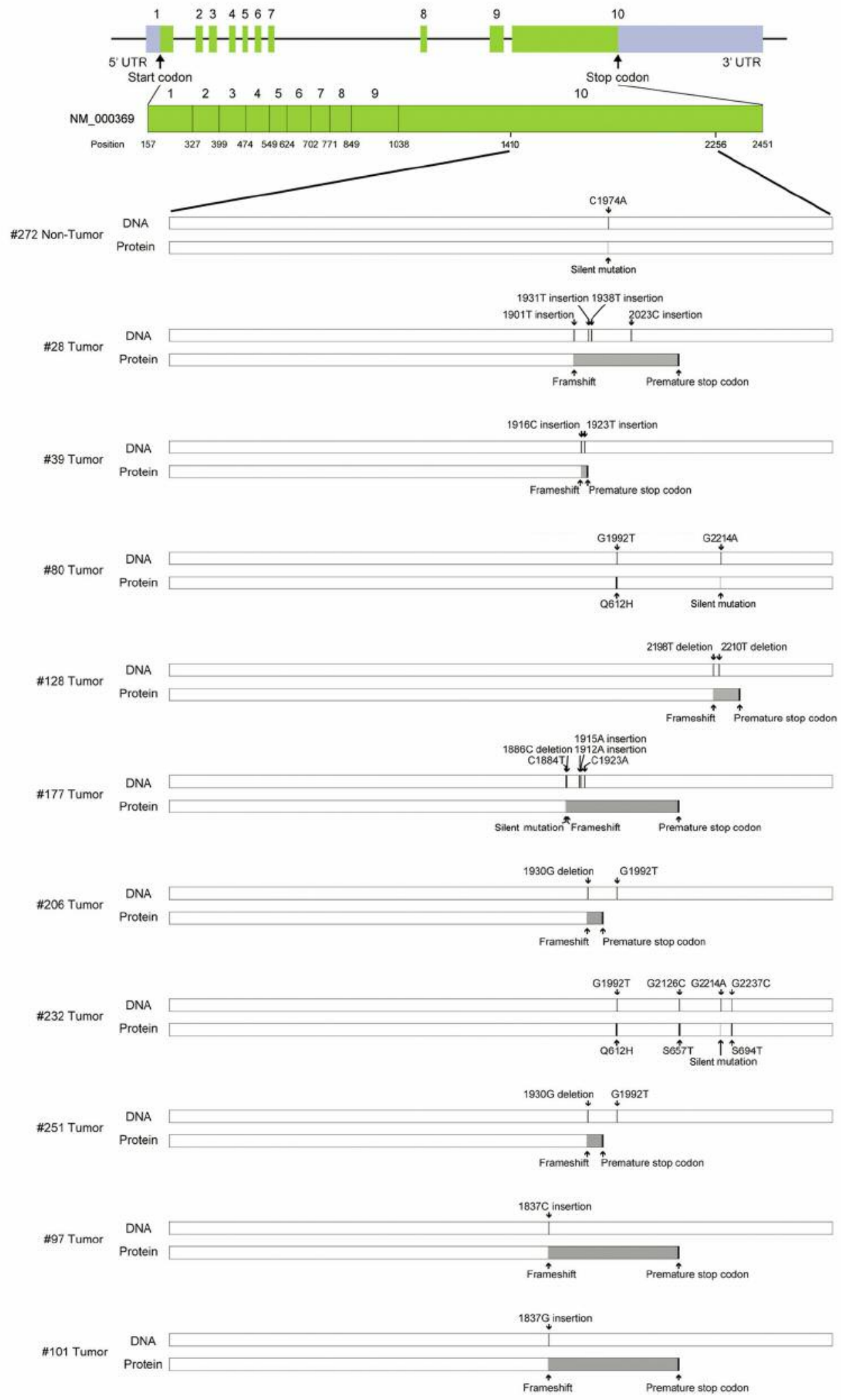

Figure 4. Detection of TSHR gene mutations in exon-10. Genomic organization of TSHR is shown in the upper two panels. The DNA and protein sequences from part of the exon-10 are depicted under them. Green boxes: exons; Blue boxes: untranslated regions (UTR); GenBank reference, NM_000369; Grey boxes: different reading frames following frame-shift mutations. 
Table III. Univariate and multivariate cox proportional hazard analysis of time-to-distant metastasis in $\mathrm{HCC}$ patients receiving surgical resection.

\begin{tabular}{lccc}
\hline Parameters & Hazard ratio & $95 \% \mathrm{CI}$ & $p$-Value \\
\hline Gender, Male & 1.324 & $0.498-3.522$ & 0.574 \\
Age, $>45$ years & 1.215 & $0.488-3.027$ & 0.675 \\
HBsAg, positive & 0.670 & $0.291-1.542$ & 0.346 \\
Anti-HCV, positive & 0.563 & $0.212-1.494$ & 0.249 \\
Cirrhosis, Yes & 0.605 & $0.279-1.309$ & 0.202 \\
Alcoholism, Yes & 0.753 & $0.284-2.001$ & 0.570 \\
Microvascular invasion, Yes & 1.605 & $0.727-3.543$ & 0.242 \\
Macrovascular invasion, Yes & 1.703 & $0.638-4.543$ & 0.287 \\
Ascites, Yes & 0.511 & $0.069-3.786$ & 0.511 \\
Histology grade, $>2$ & 1.215 & $0.527-2.799$ & 0.648 \\
Capsule, Yes & 0.775 & $0.345-1.741$ & 0.537 \\
Tumor number, $>1$ & 0.902 & $0.415-1.957$ & 0.794 \\
Tumor size, $>6$ cm & 3.160 & $1.456-6.858$ & 0.004 \\
Biochemistry & & & \\
AFP, $>30$ ng/ml & 1.907 & $0.859-4.232$ & 0.113 \\
Albumin, $>3.9$ g/dl & 0.253 & $0.109-0.585$ & 0.001 \\
Bilirubin, $>1.1 \mathrm{mg} / \mathrm{dl}$ & 1.519 & $0.660-3.495$ & 0.326 \\
Prothrombin time, $>12$ sec & 1.053 & $0.483-2.293$ & 0.897 \\
AST, $>39$ U/1 & 1.003 & $0.460-2.186$ & 0.993 \\
ALT, $>42$ U/1 & 0.653 & $0.291-1.466$ & 0.302 \\
Creatinine, $>1$ mg/dl & 0.415 & $0.190-0.904$ & 0.027 \\
TSHR & & & \\
TSHR_N, $>110$ HU & 0.718 & $0.288-1.791$ & 0.477 \\
TSHR_T, $>115$ HU & 2.927 & $1.008-8.500$ & 0.048 \\
TSHR_T/N ratio, >2.4 & 2.438 & $1.060-5.610$ & 0.036 \\
\hline
\end{tabular}

Multivariate analysis (Entry mode)

\begin{tabular}{lccc}
\hline Parameters & Hazard ratio & $95 \% \mathrm{CI}$ & $p$-Value \\
\hline Tumor size, $>6 \mathrm{~cm}$ & 1.767 & $0.741-4.212$ & 0.199 \\
Albumin, $>3.9 \mathrm{~g} / \mathrm{dl}$ & 0.299 & $0.126-0.712$ & 0.006 \\
Creatinine, $>1 \mathrm{mg} / \mathrm{dl}$ & 0.544 & $0.233-1.275$ & 0.161 \\
TSHR_T, $>115 \mathrm{HU}$ & 2.169 & $0.610-7.704$ & 0.231 \\
TSHR_TN ratio, $>2.4$ & 1.512 & $0.568-4.026$ & 0.408 \\
\hline
\end{tabular}

\section{Discussion}

In cancers other than HCC, thyroid hormone is considered a growth-promoting factor for the tumor, while hypothyroidism is associated with a better clinical prognosis $(12,13,20)$. In HCC, however, hypothyroidism has been proposed to be a risk factor (14). Patients with hypothyroidism would have a reciprocally higher level of TSH. Conceivably, the TSH-TSHR signaling would affect HCC cell properties if HCC expresses TSHR. In HCC patients receiving surgical treatment, high TSHR expression was correlated with unfavorable postoperative outcomes. We speculate that TSHR-mediated signaling possibly results in growth promotion of HCCs. TSHR belongs to the G-protein-
Table IV. Linear regression analysis for clinical parameters associated with TSHR expression levels in HCC cancerous parts.

\begin{tabular}{lrcc}
\hline Parameters & Beta & 95\%CI & $p$-Value \\
\hline Gender, Male & 71.398 & -52.564 to 195.360 & 0.257 \\
Age, years & -1.477 & -5.064 to 2.110 & 0.418 \\
HBsAg, positive & 0.242 & -117.141 to 117.626 & 0.997 \\
Anti-HCV, positive & -54.266 & -165.528 to 56.996 & 0.337 \\
Cirrhosis, Yes & -53.552 & -157.810 to 50.706 & 0.312 \\
Alcoholism, Yes & -27.581 & -143.252 to 88.090 & 0.639 \\
Microvascular invasion, Yes & 82.634 & -24.864 to 190.133 & 0.131 \\
Macrovascular invasion, Yes & 66.604 & -68.708 to 201.917 & 0.333 \\
Ascites, Yes & 0.795 & -177.377 to 178.967 & 0.993 \\
Histology grade & 88.381 & 12.392 to 164.370 & 0.023 \\
Capsule, Yes & -30.596 & -144.018 to 82.827 & 0.595 \\
Tumor number & 32.506 & -10.860 to 75.872 & 0.141 \\
Tumor size, cm & 13.326 & 0.506 to 26.147 & 0.042 \\
Biochemistry & & & \\
AFP, ng/ml & 0.003 & 0.001 to 0.004 & $<0.001$ \\
Albumin, g/dl & 38.667 & -48.914 to 126.249 & 0.385 \\
Bilirubin, mg/dl & -9.687 & -43.076 to 23.702 & 0.568 \\
Prothrombin time, sec & 0.810 & -5.617 to 7.236 & 0.804 \\
AST, U/l & 0.005 & -0.556 to 0.566 & 0.986 \\
ALT, U/l & -0.034 & -0.413 to 0.344 & 0.858 \\
Creatinine, mg/dl & -14.162 & -64.166 to 35.842 & 0.577 \\
\hline Multivariate analysis & & & \\
\hline Histology grade & 76.130 & 0.525 to 151.735 & 0.048 \\
Tumor size, cm & 7.635 & -5.277 to 20.547 & 0.245 \\
AFP, ng/ml & 0.003 & 0.001 to 0.004 & 0.001 \\
\hline & & & \\
\hline
\end{tabular}

coupled receptor family. As with other G-protein-coupled receptors, activation of TSH-TSHR signaling results in cAMP elevation (34), which in turn could activate MAPK pathways (35). In the immunohistochemical analysis, we discovered that TSHR was localized in the nuclei/peri-nuclei of HCC cells in some patients (Figure 1B). Several other studies have also reported that other G-protein-coupled receptors could localize to the cell nuclei $(36,37)$. A recent study showed that amino acid polymorphisms in G-protein coupled estrogen receptor results in topological changes, perturbing protein glycosylation and resulting in nuclear translocation of the receptor (38).

Mutation analysis revealed that $T S H R$ gene mutations leading to TSHR truncation could be found in $8 / 81$ of cancerous tissues but not in noncancerous tissues, suggesting a growth advantage of cancer cells carrying such mutations. Recent studies indicated that truncated TSHR could form a heterodimer with wild-type TSHR and thus increased their ER or Golgi retention $(39,40)$. Furthermore, it was found that internalized TSH-TSHR from the cell membrane could trigger a more persistent signaling (3941). It is, therefore, reasonable to speculate that the truncated TSHRs served a role to prolong the signaling elicited from the 
internalized TSH-TSHR heterodimers. At this time, it is still debatable whether the nature of such signaling is the same as that from the cell membrane.

Another important biological change following TSH stimulation was the alterations in cell sensitivity to anticancer agents. It was found that the sensitivity to cisplatin could be either increased, decreased, or remain unchanged upon TSH stimulation. Whether the alteration in drug sensitivity upon TSH stimulation can be applied to clinical use still awaits further investigation.

In summary, we discovered that TSHR was overexpressed in a great majority of HCC tissues. These TSHRs were deemed to be functional as in vitro stimulation by TSH resulted in elevation of cellular cAMP and alteration of cell sensitivity to an anticancer agent. Furthermore, high expression of TSHR was associated with an unfavorable postoperative outcome in early-stage HCC patients.

\section{Acknowledgements}

The Authors appreciate the help of the members of the Liver Research Center, Chang Gung Memorial Hospital for the critical discussion and assembly of all clinical data. This work was supported by the Chang Gung Medical Research Program (CORPG3G0971 and CMRPG1B0571).

\section{References}

1 Ferlay J, Soerjomataram I, Dikshit R, Eser S, Mathers C, Rebelo M, Parkin DM, Forman D and Bray F: Cancer incidence and mortality worldwide: sources, methods and major patterns in GLOBOCAN 2012. Int J Cancer 136: E359-386, 2015.

2 Bosetti C, Turati F and La Vecchia C: Hepatocellular carcinoma epidemiology. Best Pract Res Clin Gastroenterol 28: 753-770, 2014.

3 Karaman B, Battal B, Sari S and Verim S: Hepatocellular carcinoma review: current treatment, and evidence-based medicine. World J Gastroenterol 20: 18059-18060, 2014.

4 Lin S, Hoffmann K and Schemmer P: Treatment of hepatocellular carcinoma: a systematic review. Liver Cancer 1: 144$158,2012$.

5 Nishikawa H, Kita R, Kimura T and Osaki Y: Transcatheter Arterial Embolic Therapies for Hepatocellular Carcinoma: A Literature Review. Anticancer Res 34: 6877-6886, 2014.

6 Yeh YP, Hu TH, Cho PY, Chen HH, Yen AM, Chen SL, Chiu SY, Fann JC, Su WW, Fang YJ, Chen ST, San HC, Chen HP, Liao CS and Changhua Community-Based Abdominal Ultrasonography Screening G: Evaluation of abdominal ultrasonography mass screening for hepatocellular carcinoma in Taiwan. Hepatology 59: 1840-1849, 2014.

7 Yen PM: Physiological and molecular basis of thyroid hormone. Physiol Rev 81: 1097-1142, 2001.

8 Hall LC, Salazar EP, Kane SR and Liu N: Effects of thyroid hormones on human breast cancer cell proliferation. J Steroid Biochem Mol Biol 109: 57-66, 2008.

9 Iishi H, Tatsuta M, Baba M, Okuda S and Taniguchi H: Enhancement by thyroxine of experimental carcinogenesis induced in rat colon by azoxymethane. Int J Cancer 50: 974-976, 1992.
10 Kress E, Skah S, Sirakov M, Nadjar J, Gadot N, Scoazec JY, Samarut $\mathbf{J}$ and Plateroti M: Cooperation between the thyroid hormone receptor TRalpha1 and the WNT pathway in the induction of intestinal tumorigenesis. Gastroenterology 138: 1863-1874, 2010.

11 Ashur-Fabian O, Blumenthal DT, Bakon M, Nass D, Davis PJ and Hercbergs A: Long-term response in high-grade optic glioma treated with medically induced hypothyroidism and carboplatin: a case report and review of the literature. Anticancer Drugs 24: 315-323, 2013.

12 Riesenbeck LM, Bierer S, Hoffmeister I, Kopke T, Papavassilis P, Hertle L, Thielen B and Herrmann E: Hypothyroidism correlates with a better prognosis in metastatic renal cancer patients treated with sorafenib or sunitinib. World J Urol 29: 807-813, 2011.

13 Nelson M, Hercbergs A, Rybicki L and Strome M: Association between development of hypothyroidism and improved survival in patients with head and neck cancer. Arch Otolaryngol Head Neck Surg 132: 1041-1046, 2006.

14 Hassan MM, Kaseb A, Li D, Patt YZ, Vauthey JN, Thomas MB, Curley SA, Spitz MR, Sherman SI, Abdalla EK, Davila M, Lozano RD, Hassan DM, Chan W, Brown TD and Abbruzzese JL: Association between hypothyroidism and hepatocellular carcinoma: a case-control study in the United States. Hepatology 49: 1563-1570, 2009.

15 Reddy A, Dash C, Leerapun A, Mettler TA, Stadheim LM, Lazaridis KN, Roberts RO and Roberts LR: Hypothyroidism: a possible risk factor for liver cancer in patients with no known underlying cause of liver disease. Clin Gastroenterol Hepatol 5: 118-123, 2007.

16 Chen RN, Huang YH, Yeh CT, Liao CH and Lin KH: Thyroid hormone receptors suppress pituitary tumor transforming gene 1 activity in hepatoma. Cancer Res 68: 1697-1706, 2008.

17 Liao CH, Yeh CT, Huang YH, Wu SM, Chi HC, Tsai MM, Tsai CY, Liao CJ, Tseng YH, Lin YH, Chen CY, Chung IH, Cheng WL, Chen WJ and Lin KH: Dickkopf 4 positively regulated by the thyroid hormone receptor suppresses cell invasion in human hepatoma cells. Hepatology 55: 910-920, 2012.

18 Huang YH, Liao CH, Chen RN, Liao CJ and Lin KH: Human testicular orphan receptor 4 enhances thyroid hormone receptor signaling. J Cell Physiol 222: 347-356, 2010.

19 Lin YH, Huang YH, Wu MH, Wu SM, Chi HC, Liao CJ, Chen CY, Tseng YH, Tsai CY, Tsai MM and Lin KH: Thyroid hormone suppresses cell proliferation through endoglin-mediated promotion of p21 stability. Oncogene 32: 3904-3914, 2013.

20 Antunes TT, Gagnon A, Bell A and Sorisky A: Thyroidstimulating hormone stimulates interleukin-6 release from 3T3L1 adipocytes through a cAMP-protein kinase A pathway. Obes Res 13: 2066-2071, 2005.

21 Sorisky A and Gagnon A: Freedom of expression beyond the thyroid: the thyroid-stimulating hormone receptor in the adipocyte. OA Biochemistry 2: 2, 2014.

22 Boutin A, Eliseeva E, Gershengorn MC and Neumann S: betaArrestin-1 mediates thyrotropin-enhanced osteoblast differentiation. FASEB J 28: 3446-3455, 2014.

23 Balzan S, Nicolini G, Forini F, Boni G, Del Carratore R, Nicolini A, Carpi A and Iervasi G: Presence of a functional TSH receptor on human erythrocytes. Biomed Pharmacother 61: 463-467, 2007.

24 Drvota V, Janson A, Norman C, Sylven C, Haggblad J, Bronnegard $\mathrm{M}$ and Marcus C: Evidence for the presence of functional thyrotropin receptor in cardiac muscle. Biochem Biophys Res Commun 211: 426-431, 1995. 
25 Kim MJ, Oh SW, Youn H, Na J, Kang KW, Park DJ, Park YJ, Jang JJ, Lee KE, Jung KC and Chung JK: Thyroid-Related Protein Expression in the Human Thymus. Int $\mathbf{J}$ Endocrinol 2017: 8159892, 2017.

26 Tian L, Song Y, Xing M, Zhang W, Ning G, Li X, Yu C, Qin C, Liu J, Tian X, Sun X, Fu R, Zhang L, Zhang X, Lu Y, Zou J, Wang L, Guan Q, Gao L and Zhao J: A novel role for thyroidstimulating hormone: up-regulation of hepatic 3-hydroxy-3methyl-glutaryl-coenzyme A reductase expression through the cyclic adenosine monophosphate/protein kinase $\mathrm{A} / \mathrm{cyclic}$ adenosine monophosphate-responsive element binding protein pathway. Hepatology 52: 1401-1409, 2010.

27 Kim JW, Lee S, Lui N, Choi H, Mulvihill M, Fang LT, Kang HC, Kwon YW, Jablons D and Kim IJ: A somatic TSHR mutation in a patient with lung adenocarcinoma with bronchioloalveolar carcinoma, coronary artery disease and severe chronic obstructive pulmonary disease. Oncol Rep 28: 1225-1230, 2012.

28 Govindaraj V, Yaduvanshi NS, Krishnamachar H and Rao AJ: Expression of thyroid-stimulating hormone receptor, octamerbinding transcription factor 4 , and intracisternal A particlepromoted polypeptide in human breast cancer tissues. Horm Mol Biol Clin Investig 9: 173-178, 2012.

29 Gyftaki R, Liacos C, Politi E, Liontos M, Saltiki K, Papageorgiou T, Thomakos N, Haidopoulos D, Rodolakis A, Alevizaki M, Bamias A and Dimopoulos A: Differential transcriptional and protein expression of thyroid-stimulating hormone receptor in ovarian carcinomas. Int J Gynecol Cancer 24: 851-856, 2014.

30 Yeh CT, Hsu CW, Chang ML and Tsao ML: Impact of the novel hepatotropic viruslike agent NV-F during chronic hepatitis $\mathrm{C}$ virus infection. J Infect Dis 198: 1742-1748, 2008.

31 Chang KW, Chou A, Lee CC, Yeh C, Lai MW, Yeh TS, Chen TC, Liang KH and Yeh CT: Overexpression of kappa-actin alters growth properties of hepatoma cells and predicts poor postoperative prognosis. Anticancer Res 31: 2037-2044, 2011.

32 Lin CL, Chien RN, Lin SM, Ke PY, Lin CC and Yeh CT: An occult hepatitis B-derived hepatoma cell line carrying persistent nuclear viral DNA and permissive for exogenous hepatitis B virus infection. PLoS one 8: e65456, 2013.

33 Nagayama Y, Chazenbalk GD, Takeshita A, Kimura H, Ashizawa K, Yokoyama N, Rapoport B and Nagataki S: Studies on homologous desensitization of the thyrotropin receptor in 293 human embryonal kidney cells. Endocrinology 135: 1060-1065, 1994.
34 Selbie LA and Hill SJ: G protein-coupled-receptor cross-talk: the fine-tuning of multiple receptor-signalling pathways. Trends Pharmacol Sci 19: 87-93, 1998.

35 Villard JD, Wicker R, Crespo P, Russo D, Filetti S, Gutkind J, Sarasin A and SuaA rez H: Role of the cAMP and MAPK pathways in the transformation of mouse $3 \mathrm{~T} 3$ fibroblasts by a TSHR gene constitutively activated by point mutation. Oncogene 19: 4896-4905, 2000.

36 Joyal JS, Bhosle VK and Chemtob S: Subcellular G-protein coupled receptor signaling hints at greater therapeutic selectivity. Expert Opin Ther Targets 19: 717-721, 2015.

37 Nielsen CK, Campbell JIA, Öhd JF, Mörgelin M, Riesbeck K, Landberg Gr and Sjölander A: A novel localization of the Gprotein-coupled CysLT1 receptor in the nucleus of colorectal adenocarcinoma cells. Cancer Res 63: 732-742, 2005.

38 Pupo M, Bodmer A, Berto M, Maggiolini M, Dietrich P-Y and Picard D: A genetic polymorphism repurposes the G-protein coupled and membrane-associated estrogen receptor GPER to a transcription factor-like molecule promoting paracrine signaling between stroma and breast carcinoma cells. Oncotarget 8 : 46728-46744, 2017.

39 Wise $\mathrm{H}$ : The roles played by highly truncated splice variants of G protein-coupled receptors. J Mol Signal 7: 13, 2012.

40 Calebiro D, Nikolaev VO, Persani L and Lohse MJ: Signaling by internalized G-protein-coupled receptors. Trends Pharmacol Sci 31: 221-228, 2010.

41 Calebiro D, Nikolaev VO, Gagliani MC, de Filippis T, Dees C, Tacchetti C, Persani L and Lohse MJ: Persistent cAMP-signals triggered by internalized G-protein-coupled receptors. PLoS Biol 7: e1000172, 2009. 